\title{
Impact of Xpert MTB/RIF and decentralized care on linkage to care and drug-resistant tuberculosis treatment outcomes in Johannesburg, South Africa
}

Denise Evans ${ }^{1 *}$ (D) Tembeka Sineke ${ }^{1}$, Kathryn Schnippel ${ }^{2}$, Rebecca Berhanu ${ }^{3}$, Caroline Govathson ${ }^{1}$, Andrew Black ${ }^{4}$, Lawrence Long ${ }^{1,5}$ and Sydney Rosen ${ }^{1,5}$

\begin{abstract}
Background: In 2011, South Africa improved its ability to test for rifampicin-resistant TB (RR-TB) by introducing GeneXpert MTB/RIF. At the same time, the South African National TB program adopted a policy decentralized, outpatient treatment for drug resistant (DR-) TB. We aim to analyze the impact of these changes on linkage to care and DR-TB treatment outcomes.

Methods: We retrospectively matched adult patients diagnosed with laboratory-confirmed RR-TB in Johannesburg from 07/2011-06/2012 (early cohort) and 07/2013-06/2014 (late cohort) with records of patients initiating DR-TB treatment at one of the city's four public sector treatment sites. We determine the proportion of persons diagnosed with RR-TB who initiated DR-TB treatment and report time to treatment initiation (TTI) before and after the implementation of Xpert MTB/RIF roll-out in Johannesburg, South Africa. We conducted a sub-analysis among those who initiated DR-TB treatment at the decentralized outpatient DR-TB centers to determine if delays in treatment initiation have a subsequent impact on treatment outcomes.

Results: Five hundred ninety four patients were enrolled in the early cohort versus 713 in the late cohort. 53.8 and $36.8 \%$ of patients were diagnosed with multi-drug resistant TB in the early and late cohorts, respectively. The proportion of RR-TB confirmed cases diagnosed by Xpert MTB/RIF increased from 43.4 to $60.5 \%$ between the early and late cohorts, respectively. The proportion who initiated treatment increased from $43.1 \%(n=256)$ to $60.3 \%$ $(n=430)$ in the late cohort. Pre-treatment mortality during the early and the late cohort reduced significantly from 17.5 to $5.8 \%$ while lost to follow-up remained high.

Although $\Pi$ TI reduced by a median of 19 days, from 33 days (IQR 12-52) in the early cohort to 14 days (IQR 7-31) in the late cohort, this did not translate to improved treatment outcomes and we found no difference in terms of treatment success or on-treatment mortality for those that initiated without delay vs. those that deferred initiation.

Conclusion: Pre-treatment mortality reduced significantly during late Xpert MTB/RIF coverage but there was no significant difference after treatment was initiated. Despite improvements there is still a significant diagnosis and treatment gap for patients diagnosed with RR-TB and improving treatment outcomes remains critical.
\end{abstract}

Keywords: Rifampicin resistance, South Africa, Time to treatment initiation, Decentralization, Xpert MTB/RIF, HIV positive, Mortality

\footnotetext{
* Correspondence: devans@heroza.org

${ }^{1}$ Health Economics and Epidemiology Research Office, Department of Internal Medicine, School of Clinical Medicine, Faculty of Health Sciences, University of the Witwatersrand, Johannesburg, South Africa

Full list of author information is available at the end of the article
}

(c) The Author(s). 2018 Open Access This article is distributed under the terms of the Creative Commons Attribution 4.0 International License (http://creativecommons.org/licenses/by/4.0/), which permits unrestricted use, distribution, and reproduction in any medium, provided you give appropriate credit to the original author(s) and the source, provide a link to the Creative Commons license, and indicate if changes were made. The Creative Commons Public Domain Dedication waiver (http://creativecommons.org/publicdomain/zero/1.0/) applies to the data made available in this article, unless otherwise stated. 


\section{Background}

The World Health Organization (WHO) estimates that only $20 \%$ of the 580,000 people eligible for treatment of multi-drug resistant and rifampicin-resistant tuberculosis (MDR/RR-TB) globally in 2015 were enrolled in care [1]. In South Africa, a high-burden country for drug resistant (DR-) TB, 64\% of the 19,613 laboratory diagnosed cases of RR-TB, including those with MDR-TB, were enrolled for treatment that year, indicating a large gap between diagnosis and treatment $[1,2]$.

Rapid diagnosis and timely treatment initiation are fundamental to control of the spread of DR-TB. To this end, the South African National TB program launched decentralized DR-TB services and the national use of Cepheid's Xpert MTB/RIF in 2011. Decentralization of services allows patients with DR-TB to be treated as outpatients, increases the number of treatment facilities, reduces cost, and improves time to treatment initiation [3-5]. Xpert MTB/RIF is a rapid molecular test that provides same day diagnosis of TB and rifampicin susceptibility. By accelerating diagnosis, Xpert MTB/RIF reduces time to treatment initiation, thereby increasing the proportion of diagnosed patients who receive treatment [2, 5]. Reducing diagnostic delays is critical to reducing primary loss to follow-up and ongoing transmission of DR-TB [6-9]. While a reduction in time to diagnosis has been reported with Xpert MTB/RIF, there is less evidence that reducing time to treatment initiation (TTI) results in better treatment outcomes.

Despite improvements in diagnostic technology and access to care via decentralization of services, many patients diagnosed with MDR/RR-TB in South Africa continue to experience delays in treatment initiation and failure to link from testing to care [10]. Estimating the number of such patients is challenging, as two unlinked data sources are required. The number of diagnosed DR-TB patients is derived from centralized laboratory records, while the number who start DR-TB treatment is obtained from TB treatment registers (paper and electronic) kept at treatment sites.

In this study we compare the proportions of patients with MDR/RR-TB who successfully linked to care in two cohorts with different levels of Xpert MTB/RIF coverage: July 2011 to June 2012 (early Xpert MTB/RIF implementation, limited coverage) and July 2013 to June 2014 (late Xpert MTB/RIF implementation, full coverage). We then evaluate whether delays in treatment initiation have a subsequent impact on treatment outcomes.

\section{Methods}

We conducted a retrospective medical register review to match adult patients diagnosed with laboratory-confirmed MDR/RR-TB, as reported to the City of Johannesburg Health Office (COJ), to treatment initiation records at the city's four public sector DR-TB treatment sites between July 2011 and June 2014. Patients who transferred into Johannesburg after starting treatment in another district, province, or country were excluded from the analysis.

\section{Defining the early and late Xpert MTB/RIF cohorts}

In order to determine the impact of Xpert MTB/RIF and decentralized DR-TB care on the proportion of persons diagnosed with RR-TB who initiated DR-TB treatment or time to treatment initiation, and whether this resulted in an improvement in DR-TB treatment outcomes, we defined two cohorts (e.g. early and late Xpert MTB/RIF implementation) and compared key $\mathrm{TB}$ indicators between the two cohorts.

South Africa's National Health Laboratory Service (NHLS) rolled-out Xpert MTB/RIF to its national network of referral laboratories between 2011 and 2013. By May/June 2012 Xpert MTB/RIF implementation was $35 \%$ complete, and by July 2013 there was full (>90\%) coverage of Xpert MTB/RIF in the study area. We divided the study population into two 12-month time periods based on specimen collection date: an early/limited access Xpert MTB/RIF cohort from July 2011 to June 2012 (hereafter referred to as "early"), and an expanded/ full access Xpert MTB/RIF cohort from July 2013 to June 2014 (hereafter referred to as "late"). The selection of these periods is consistent with what has been used in previous reports [2], and supported by data on Xpert MTB/RIF coverage from the NHLS for the COJ (Additional file 1: Figure S1).

\section{Data collection}

The COJ Health Office, using a team of sub-district TB coordinators, coordinates the DR-TB response throughout the municipality, which includes both the central city and a large number of suburban neighborhoods and informal settlements surrounding it and has a population of approximately 4.4 million. The DR-TB response includes treatment at three decentralized (outpatient) hospital-based clinics and one centralized (inpatient) DR-TB hospital, according to the South African National TB guidelines $[4,11]$. TB test results with indication of rifampicin drug resistance are communicated from the NHLS to the COJ where the patient is entered into a central electronic COJ register, alongside a DR-TB case registration number. The register captures first name, last name, date of birth, sex, tracing outcome, disease classification, diagnosis method, date of specimen collection, smear microscopy status, and patient address.

We identified our cohorts from the COJ register based on the specimen collection date and used NHLS data to verify the diagnosis method and specimen collection date. We enrolled all adult patients (18 years and older) who were diagnosed between July 2011-June 2012 or 
between July 2013-June 2014 and assigned a DR-TB case registration number by the $\mathrm{COJ}$.

We then matched patients from the COJ register to the treatment registers at the four DR-TB treatment sites (Fig. 1). Details of the matching and the validation of the matching algorithm have been described before [3]. Treatment start dates were obtained from treatment registers. For patients who were treated at the decentralized outpatient DR-TB centers, we also recorded the final treatment outcome assigned in the treatment registers, which allowed us to conduct a sub-analysis of outcomes as described below. Patients were followed for at least 24 months from the date of treatment initiation. Study staff worked closely with facilities and the COJ to verify and correct patient information (e.g. to correctly record DR-TB registration numbers in facility registers and query specific cases where diagnosis date or treatment initiation date preceded the sputum collection date) [3]. Medical records could only be queried for patients who initiated DR-TB treatment at one of the city's four treatment sites. Patient information for those who did not initiate DR-TB treatment at one of the city's four treatment sites could not be checked (e.g. at the diagnosing/ referring facility).

\section{Classification of drug-resistance patterns}

Patients diagnosed with rifampicin resistance by Xpert MTB/RIF with unknown susceptibility to isoniazid or second-line TB drugs were classified as having rifampicin resistant TB (RR-TB). When susceptibility test results for isoniazid and second-line drugs were available, patients were categorized as either rifampicin mono-resistant (RMR), multi-drug resistant (MDR-TB) (i.e. resistant to rifampicin and isoniazid) or extensively drug resistant (XDR-TB) (i.e. resistant to rifampicin, isoniazid and second-line TB drugs) $[11,12]$.

\section{Outcomes \\ Linkage to care}

The primary outcome of interest was the proportion of patients diagnosed with MDR/RR-TB who initiated DR-TB treatment at one of the city's four treatment sites within 6 months of specimen collection. Patients were followed from the specimen collection date until the earliest of treatment initiation (linkage), all-cause mortality, loss to care, transfer or relocation out of the city, or 6 months' follow-up. Possible outcomes in this linkage to care analysis included death before linkage, moved or transferred out of the city, lost to follow-up (untraceable), failed to link to care or successfully traced and referred for treatment.

Descriptive statistics (frequencies, medians, and interquartile ranges) were used to present demographic and clinical characteristics present at diagnosis. The diagnosis method and diagnosis date were extracted from the $\mathrm{COJ}$ register and we present the median time from specimen collection to diagnosis date.

\section{Impact of time to treatment initiation on final outcome of treatment}

We defined time to treatment initiation as the difference between the specimen collection date and the date that DR-TB treatment was initiated. We compared median time to treatment initiation by disease classification (i.e. RR-TB, RMR, MDR-TB or XDR-TB) and diagnosis method using the student $t$ test for parametric or Kruskal-Wallis for non-parametric data. Using the time to treatment initiation and the diagnostic method used, treatment initiation was categorized as delayed (deferred) or without delay (Table 1). In the sub-study, we also analyzed the impact of time to treatment initiation on final outcomes of treatment.

\section{Final treatment outcomes}

For the sub-study among patients who initiated DR-TB treatment at the three decentralized outpatient DR-TB centers, DR-TB outcomes were assigned in the case registers according to standard WHO definitions and 2013 WHO Reporting Framework for Tuberculosis as treatment success (cured and completed), failed, lost to follow-up, died, or transfer to another facility [12, 13]. Data from patients who transferred out or did not have an outcome assigned (i.e. missing) were excluded from this analysis, as no final treatment outcomes were available.

We identified patient characteristics at DR-TB initiation associated with treatment success (24 months after the DR-TB treatment start date) using log-binomial regression to estimate the relative risk and 95\% confidence interval. We also identified predictors of on-treatment, all-cause mortality using Cox proportional hazards to estimate the hazard ratio and 95\% confidence interval. Patients were followed from the DR-TB treatment start date until the earliest of all-cause mortality, lost to follow-up, treatment failure, treatment failure or 24 months' follow-up. Variables in the univariate model that were significant at the 0.25 level along with other a priori identified characteristics (e.g. age, HIV status, gender etc.) were included in the multivariate model. The univariate and multivariate (adjusted) results are presented. Patients who initiated or were later referred for treatment at Sizwe Tropical Diseases Hospital, the centralized DR-TB inpatient treatment site for the province, were not included in this analysis as they had more extensive resistance (e.g. XDR or pre-XDR) or other reasons rendering them ineligible for decentralized DR-TB care. 


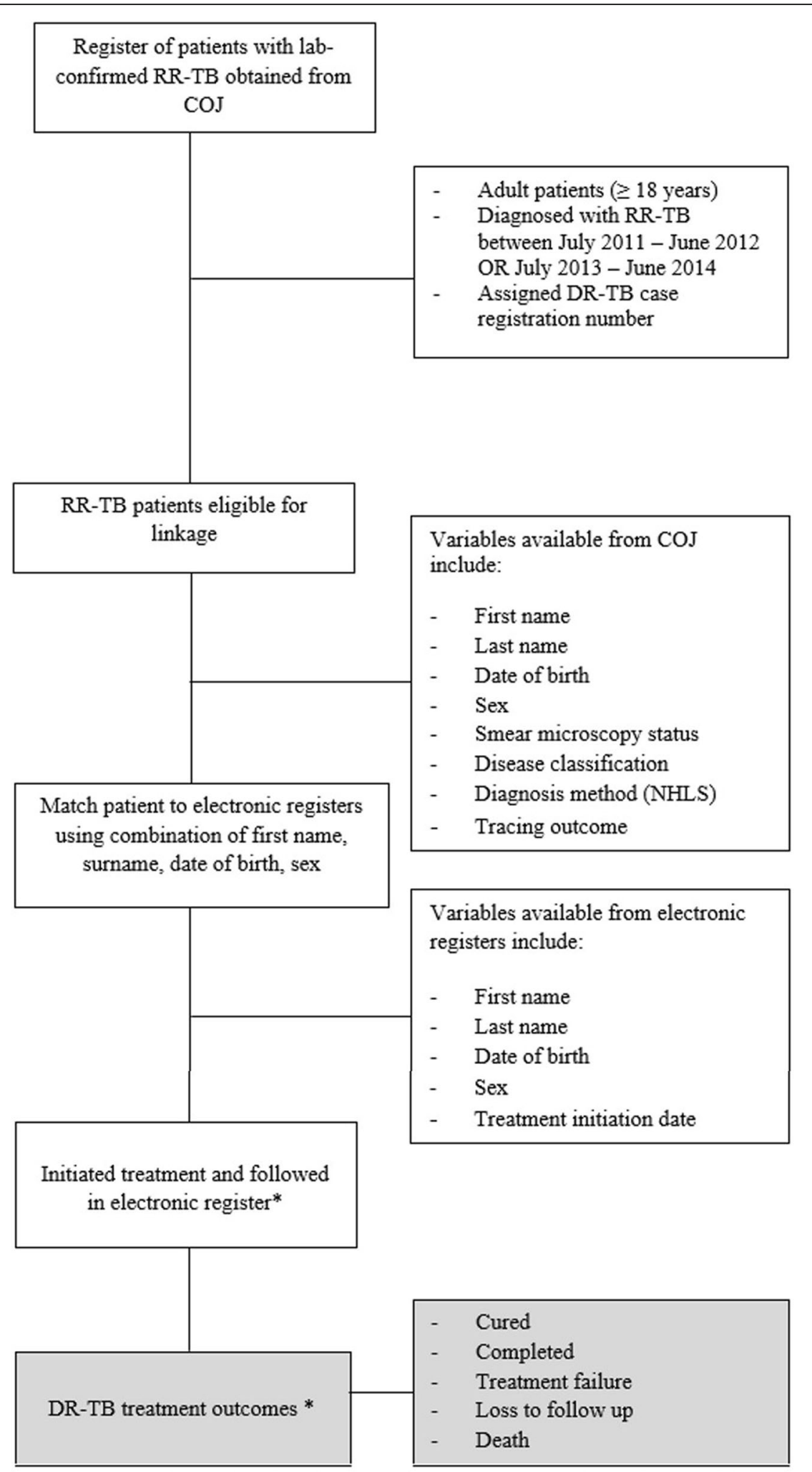

*only among patients who initiated DR-TB at the decentralized outpatient DR-TB centres

Fig. 1 Schematic illustrating inclusion and retrospective follow-up of patients diagnosed with laboratory-confirmed RR-TB, as reported to the City of Johannesburg 
Table 1 Treatment initiation categorized as delayed (deferred) or without delay according to time to treatment initiation and diagnosis method

\begin{tabular}{lll}
\hline Diagnosis method & Without delay & Delayed (deferred) \\
\hline Xpert MTB/RIF & $\leq 7$ days & $>7$ days \\
Line probe assay (LPA) & & \\
Positive smear & $\leq 24$ days & $>24$ days \\
Negative/unknown & $\leq 60$ days & $>60$ days \\
$\begin{array}{l}\text { Phenotypic drug sensitivity } \\
\text { testing (DST) }\end{array}$ & $\leq 80$ days & $>80$ days \\
\hline
\end{tabular}

All analyses were carried out using SAS version 9.3 (SAS Institute, Cary, North Carolina, USA). The study was a retrospective register review of routinely captured records and a waiver of informed consent was granted to retrospectively review these records. This study and the analysis of anonymized data was approved by the Human Research Ethics Committee (Medical) of the University of the Witwatersrand (Wits HREC M130601).

\section{Results}

A total of 594 patients were included in the early cohort and 713 patients in the late cohort. The proportion of RR-TB patients diagnosed by Xpert MTB/RIF increased from $43.4 \%$ during the early cohort to $60.5 \%$ during the late cohort, with the remaining $33.9 \%$ diagnosed by line probe assay (LPA) despite guidelines calling for resistance to be identified by Xpert MTB/RIF. Most (62.6 and 76.4\% for the early and late cohorts, respectively) patients did not have a smear microscopy result reported (Table 2).

Patients in the late cohort were slightly older and more likely to be male than those in the early cohort (median age of 37 years IQR $30-44$ and $55.6 \%$ in the late cohort vs. 34 years IQR $29-42$ and $51.6 \%$ in the early cohort). HIV prevalence in our early and late cohorts was lower than has been described nationally, with HIV prevalence of $26.1 \%$ in the early cohort vs. a national prevalence of $53.2 \%$ [14] and $47.1 \%$ in the late cohort vs. national 66\% [2]. This may be due to the high number of patients in our cohorts with a HIV status missing, reflecting the limitations of routine data use $[5,14]$.

\section{Treatment referral and initiation}

The total number of cases diagnosed at the four study sites increased from 594 in the early period to 713 in the late period, representing a $20 \%$ increase. In the early cohort, $60.9 \%(362 / 594)$ were successfully traced by COJ and referred for treatment. This increased to $69.9 \%(499 / 713)$ in the late cohort (Fig. 2). The proportion of patients diagnosed with RR-TB in COJ who initiated treatment at one of the four treatment sites within 6 months of diagnosis increased from $43.1 \%(256 / 594)$ in the early period to $60.3 \%$
(430/713) in the late period. The proportion who initiated at a decentralized (outpatient) TB facility, rather than the centralized inpatient facility, increased from 65.6 to $87.4 \%$ in the early and late cohorts, respectively.

\section{Time to diagnosis and treatment initiation}

Median time from specimen collection to diagnosis decreased considerably from 26 days (IQR 7-36) in the early cohort to 2 days (IQR 1-7) in the late cohort. Median time from specimen collection to treatment initiation fell from 33 days (IQR 12-52) during the early cohort to 14 days (IQR 7-31) during the late cohort. Decreases in time to initiation varied by diagnostic test and disease classification, as shown in Table 3. The median time to treatment initiation dropped from 17 days (IQR 9-46) to 13 days (IQR 7-28) for patients diagnosed with Xpert MTB/RIF and from 38 days (IQR 23-51) to 15 days (IQR 7-36) for those diagnosed by LPA. The percentage of diagnosed patients who initiated treatment within 5 days, which is the national target increased from $6 \%(15 / 256)$ in the early cohort to $19 \%(82 / 430)$ in the late cohort [15].

\section{Linkage to care and treatment initiation}

Death before treatment initiation decreased from $17.5 \%(n=104)$ in the early cohort to $5.8 \%(n=41)$ in the late cohort; median time from specimen collection to death was 19 days (IQR 10-30) and 29 days (IQR 12-36) in these cohorts, respectively. Loss to follow up remained high at 17.5 and $22.4 \%$, though the proportion of patients who were successfully traced but then failed to link to care decreased from 17.8 to $9.7 \%$ (Fig. 2). Median time from specimen collection to other reported outcome (lost to follow-up/ untraceable or transferred out of the city/moved) was 14 days (IQR $8-34, n=44$ ) for the early and 24 days (IQR 9-57, $n=34$ ) for the late cohort, respectively.

\section{Sub-study of outcomes and patient characteristics}

Five hundred thirty seven patients initiated treatment at one of the three decentralized facilities, a number that excludes those who initiated at the centralized inpatient facility, transferred out, did not have an outcome assigned (i.e. missing) or had more extensive resistance (i.e. XDR or pre-XDR TB). Roughly half were $30-45$ years old $(52.5 \%)$ and male $(51.9 \%)$, and $62.8 \%$ were diagnosed by Xpert MTB/RIF. Of those who initiated treatment, $79.7 \%(428 / 537)$ were HIV positive, with most on ART $(82.0 \%, 351 / 428)$, which was comparatively higher than that reported for the early and late cohorts and reflects the limitations of using routine data from two unlinked data sources (e.g. $42-70 \%$ of patients in the COJ register had an 
Table 2 Demographic and clinical characteristics of patients who had a diagnosis of laboratory-confirmed RR-TB reported to the COJ for tracing between July 2011-June $2012(n=594)$ and July 2013-June $2014(n=713)$ and those included in the treatment outcomes sub-study $(n=537)$

\begin{tabular}{|c|c|c|c|c|}
\hline Characteristic & & Early $(n=594)$ & Late $(n=713)$ & $\begin{array}{l}\text { Included in } \\
\text { sub-analysis }(n=537)\end{array}$ \\
\hline Gender & $n, \%$ & & & \\
\hline Male & & $307(51.6)$ & $397(55.6)$ & $279(51.9)$ \\
\hline Female & & $287(48.3)$ & $316(44.3)$ & $258(48.0)$ \\
\hline Age, years & Median, IQR & $34(29-42)$ & $37(30-44)$ & $36(29-43)$ \\
\hline$<30$ & & $162(27.3)$ & $161(22.6)$ & $138(25.7)$ \\
\hline $30-45$ & & $308(51.9)$ & $376(52.7)$ & $282(52.5)$ \\
\hline $45-60$ & & $105(17.6)$ & $159(22.3)$ & $106(19.7)$ \\
\hline$\geq 60$ & & $19(3.2)$ & $17(2.4)$ & $11(2.1)$ \\
\hline DR-TB classification & $n, \%$ & & & \\
\hline RR-TB by Xpert MTB/RIF & & $158(26.6)$ & $316(44.3)$ & $237(44.1)$ \\
\hline RIF mono-resistant TB & & $102(17.2)$ & $130(18.2)$ & $109(20.3)$ \\
\hline MDR-TB & & $320(53.8)$ & $262(36.8)$ & $191(35.6)$ \\
\hline XDR-TB & & $14(2.4)$ & $5(0.7)$ & $\mathrm{n} / \mathrm{a}$ \\
\hline Sputum collection to diagnosis, days & Median, IQR & $26(7-36)$ & $2(1-7)$ & $13(7-28)$ \\
\hline Diagnosis method from the COJ register & $n, \%$ & & & \\
\hline Xpert MTB/RIF & & $258(43.4)$ & $431(60.5)$ & $337(62.8)$ \\
\hline Geno Type MTBDR plus line probe assay & & $281(47.3)$ & $242(33.9)$ & $172(32.0)$ \\
\hline Phenotypic drug sensitivity testing & & $30(5.1)$ & $2(0.3)$ & $6(1.1)$ \\
\hline Unknown & & $25(4.2)$ & $38(5.3)$ & $22(4.1)$ \\
\hline AFB smear microscopy & $n, \%$ & & & \\
\hline Positive & & $144(24.3)$ & $119(16.7)$ & $94(17.4)$ \\
\hline Negative & & $78(13.1)$ & $49(6.9)$ & $44(8.2)$ \\
\hline Unknown & & $372(62.6)$ & $545(76.4)$ & $400(74.4)$ \\
\hline HIV status at initiation & $n, \%$ & & & \\
\hline Positive & & $155(26.1)$ & $336(47.1)$ & $428(79.7)$ \\
\hline HIV positive on ART & & $\mathrm{n} / \mathrm{a}$ & n/a & $351 / 428(82.0)$ \\
\hline HIV positive not on ART & & $\mathrm{n} / \mathrm{a}$ & $\mathrm{n} / \mathrm{a}$ & $77 / 428(17.9)$ \\
\hline HIV Negative & & $24(4.0)$ & $78(10.9)$ & $61(11.4)$ \\
\hline Unknown & & $415(69.9)$ & $299(42.0)$ & $48(8.9)$ \\
\hline Time on ART, months & Median, IQR & & & \\
\hline ART initiation before TB treatment initiation $(n=171)$ & & $\mathrm{n} / \mathrm{a}$ & $\mathrm{n} / \mathrm{a}$ & $9.6(3.1-27.4)$ \\
\hline ART initiation after TB treatment initiation $(n=117)$ & & $\mathrm{n} / \mathrm{a}$ & $\mathrm{n} / \mathrm{a}$ & $0.5(0.4-0.9)$ \\
\hline
\end{tabular}

MDR-TB Multi-drug resistant, XDR-TB Extensively drug resistant TB, RR-TB Rifampicin resistant TB, AFB Acid fast bacilli, $A R T$ antiretroviral therapy, $D R$ - TB drug resistant TB, RIF rifampicin

unknown HIV status compared to $<10 \%$ in the DR-TB treatment register) (Table 2).

Outcomes could be evaluated for $62.8 \%(161 / 256)$ of patients who initiated during the early Xpert MTB/ RIF period and $84.9 \%(365 / 430)$ of those who initiated in the late Xpert MTB/RIF period. Treatment success was similar between the two periods (32.9\% vs. $39.6 \%$ for the early and expanded Xpert MTB/RIF cohorts, respectively). Despite decentralization and Xpert
MTB/RIF implementation, all-cause on-treatment mortality $(11.8 \%$ vs. $14.2 \%)$ and loss to follow-up (55.3\% vs. 55.9\%) remained high for both the early and late cohorts (Fig. 2).

The only factor associated with treatment success was pattern of drug resistance (RR-TB by Xpert MTB/RIF vs. MDR-TB; aRR 4.31 95\% CI 2.55-7.29 and RMR vs. MDR-TB; aRR 4.46 95\% CI 2.07-7.95) (Table 4). There was no association between time to treatment initiation 

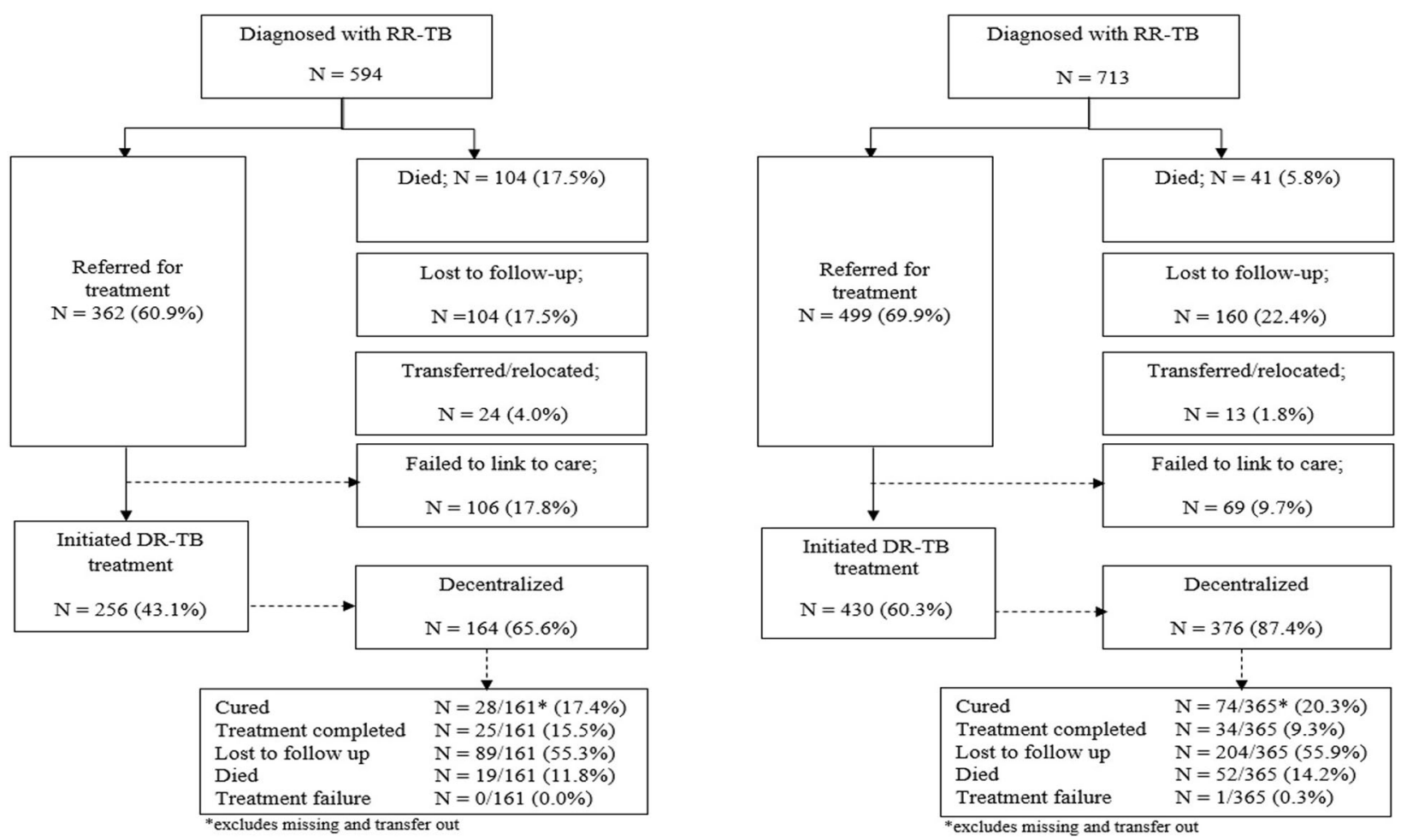

Fig. 2 Treatment referral and initiation of patients who had a diagnosis of laboratory-confirmed RR-TB reported to the COJ for tracing between July 2011-June $2012(n=594)$ and July 2013-June $2014(n=713)$

(i.e. delayed treatment initiation vs. without delay) and treatment success.

We also identified predictors of all-cause mortality after DR-TB treatment initiation (Table 5). Compared to HIV positive patients on ART, HIV negative patients were less likely to die during the 24 months of follow-up on treatment (aHR 0.13 95\% CI 0.02-0.10). Delayed (deferred) treatment initiation was not identified as a predictor of all-cause mortality (delayed vs. without delay; aHR 0.91 95\% CI 0.53-1.56).

\section{Discussion}

Tuberculosis continues to kill more people worldwide than any other infectious disease [16]. The Global Burden of Disease study 2015 concludes that strengthening health systems for early TB case detection and improved quality of diagnosis and treatment are essential to addressing this epidemic [17]. In two cohorts of DR-TB patients diagnosed in Johannesburg, South Africa in 2011 and 2013, we found that the introduction of Xpert MTB/RIF was associated with a substantial decrease in

Table 3 Median time from specimen collection to treatment initiation, by diagnostic method and disease classification $(n=686)$

\begin{tabular}{lll}
\hline & Early cohort $(n=256)$ & Late cohort $(n=430)$ \\
\hline $\begin{array}{l}\text { Diagnosis method } \\
\text { Xpert MTB/RIF }\end{array}$ & $17(9-46)(n=107)$ & $13(7-28)(n=282)$ \\
$\begin{array}{l}\text { GenoType MTBDRplus line probe assay } \\
\text { Phenotypic drug susceptibility testing }\end{array}$ & $38(23-51)(n=129)$ & $15(7-36)(n=129)$ \\
Disease classification & $81(28-97)(n=7)$ & $n / a$ \\
RR-TB by Xpert MTB/RIF & $13(9-30)(n=57)$ & $10(7-17)(n=188)$ \\
RIF mono-resistant TB & $48(30-75)(n=48)$ & $13(2-38)(n=62)$ \\
MDR-TB & $34(14-49)(n=147)$ & $22(8-37)(n=128)$ \\
XDR-TB & $42(35-70)(n=3)$ & $36(14-61)(n=4)$
\end{tabular}


Table 4 Factors associated with treatment success by 24 months among patients who were diagnosed with RR-TB between July 2011-June 2012 or between July 2013-July 2014 and who initiated DR-TB treatment in COJ ( $n=537)$

\begin{tabular}{|c|c|c|c|c|c|c|}
\hline & $\mathrm{n} / \mathrm{N}$ & $\%$ & $\mathrm{RR}$ & $95 \% \mathrm{Cl}$ & Adjusted RR & $95 \% \mathrm{Cl}$ \\
\hline \multicolumn{7}{|l|}{ Gender } \\
\hline Female & $75 / 258$ & 29.1 & Ref & Ref & Ref & Ref \\
\hline Male & $86 / 279$ & 30.8 & 1.09 & $(0.79-1.49)$ & 1.01 & $(0.74-1.41)$ \\
\hline \multicolumn{7}{|l|}{ Age, years } \\
\hline$<30$ & $31 / 138$ & 22.5 & Ref & Ref & Ref & Ref \\
\hline $30-45$ & $93 / 282$ & 32.9 & 1.56 & $(1.02-2.37)$ & 1.46 & $(0.94-2.25)$ \\
\hline$\geq 45$ & $37 / 117$ & 31.6 & 1.46 & $(0.89-2.38)$ & 1.43 & $(0.86-2.37)$ \\
\hline \multicolumn{7}{|l|}{ DR-TB classification } \\
\hline RR-TB by Xpert MTB/RIF & $96 / 237$ & 40.5 & 4.36 & $(2.60-7.30)$ & 4.31 & $(2.55-7.29)$ \\
\hline RIF mono-resistant TB & $47 / 109$ & 43.1 & 4.76 & $(2.70-8.36)$ & 4.46 & $(2.07-7.95)$ \\
\hline MDR-TB & 18/191 & 9.4 & Ref & Ref & Ref & Ref \\
\hline \multicolumn{7}{|l|}{ HIV status at initiation } \\
\hline HIV negative & $14 / 61$ & 22.1 & 0.58 & $(0.32-1.05)$ & 0.75 & $(0.47-1.20)$ \\
\hline HIV positive on ART & $120 / 351$ & 33.4 & Ref & Ref & Ref & Ref \\
\hline HIV positive not on ART & $16 / 77$ & 19.5 & 0.6 & $(0.36-1.02)$ & 0.73 & $(0.43-1.25)$ \\
\hline HIV status unknown & $11 / 48$ & 22.9 & 0.64 & $(0.33-1.21)$ & 0.76 & $(0.43-1.24)$ \\
\hline \multicolumn{7}{|l|}{ Registration year } \\
\hline 2011 & $19 / 55$ & 34.5 & Ref & Ref & Ref & Ref \\
\hline 2012 & $33 / 108$ & 30.6 & 0.88 & $(0.50-1.56)$ & 0.88 & $(0.49-1.56)$ \\
\hline 2013 & $49 / 166$ & 29.5 & 0.81 & $(0.47-1.37)$ & 0.74 & $(0.43-1.28)$ \\
\hline 2014 & $60 / 211$ & 28.4 & 0.84 & $(0.50-1.41)$ & 0.83 & $(0.49-1.41)$ \\
\hline \multicolumn{7}{|l|}{ Time to treatment initiation } \\
\hline Without delay & $70 / 186$ & 37.6 & Ref & Ref & Ref & Ref \\
\hline Delayed (deferred) & $85 / 332$ & 25.6 & 0.97 & $(0.71-1.33)$ & 0.94 & $(0.66-1.33)$ \\
\hline
\end{tabular}

$R R$ Relative Risk, $C l$ Confidence interval, RR-TB Rifampicin resistant TB, MTB Mycobacterium tuberculosis, MDR-TB Multi-drug resistant tuberculosis, XDR-TB Extensively drug-resistant tuberculosis; $D R$-TB drug resistant TB, ART antiretroviral therapy, RIF rifampicin $p<0.05$ bold

time to treatment initiation and with an increase in the proportion of patients who linked to care and initiated appropriate DR-TB treatment $[2,5]$. Our study demonstrated an additional benefit, observed during the late period, which was a significant reduction in pre-treatment mortality.

We found that the number of people diagnosed with DR-TB within the COJ between 2001 and 2012 and 2013-2014 who initiated appropriate treatment within 6 months of diagnosis increased from 43.1 to $60.3 \%$. Despite this large increase, some $40 \%$ of patients with a reported diagnosis of RR- or MDR-TB did not start treatment within 6 months, at least within the City of Johannesburg municipality. While a small proportion likely did start treatment outside the city, there remained a major gap in moving patients from diagnosis to treatment, despite the policy changes.

The increase in treatment initiation is similar to what was observed in a nationwide retrospective cohort, which reported 55 and $63 \%$ in 2011 and 2013 respectively $[2,5]$ and possibly reflects the national strategy for Xpert MTB/RIF implementation across districts or provinces, under which priority for pilot implementation or research sites in high burden districts. Our results also showed that the proportion of patients initiating appropriate DR-TB treatment within 5 days of being diagnosed increased from 6 to 19\%, still four fifths of patients were not managed according to the National Department of Health recommendation to initiate DR-TB treatment within 5 days of being diagnosed [15].

Overall, time to treatment initiation decreased from a median of 33 to 14 days in the early and late cohorts, respectively. Among patients diagnosed by Xpert MTB/ RIF, time to treatment initiation decreased from 17 to 13 days. Our observed time to treatment initiation is much lower than that reported by a national cohort study based on data from the national RR-TB register, which was 44 and 22 days [2]. This may reflect true variation by geographic region in South Africa or simply limitations of the national register, as only $53 \%$ of the 
Table 5 Unadjusted and adjusted predictors of mortality among patients who were diagnosed with RR-TB between July 2011-June 2012 or between July 2013-July 2014 and who initiated DR-TB treatment in COJ $(n=537)$

\begin{tabular}{|c|c|c|c|c|c|c|}
\hline & $\mathrm{n} / \mathrm{N}$ & $\%$ & $\mathrm{HR}$ & $95 \% \mathrm{Cl}$ & Adjusted HR & $95 \% \mathrm{Cl}$ \\
\hline \multicolumn{7}{|l|}{ Gender } \\
\hline Female & $34 / 258$ & 13.2 & Ref & Ref & Ref & Ref \\
\hline Male & $33 / 279$ & 11.8 & 0.78 & $(0.48-1.26)$ & 0.74 & $(0.44-1.22)$ \\
\hline \multicolumn{7}{|l|}{ Age, years } \\
\hline$<30$ & $17 / 138$ & 12.3 & Ref & Ref & Ref & Ref \\
\hline $30-45$ & $37 / 283$ & 13.1 & 0.92 & $(0.52-1.64)$ & 0.72 & $(0.39-1.31)$ \\
\hline$\geq 45$ & $13 / 119$ & 10.9 & 0.75 & $(0.36-1.58)$ & 0.68 & $(0.32-1.47)$ \\
\hline \multicolumn{7}{|l|}{ DR-TB classification } \\
\hline RR-TB by Xpert MTB/RIF & $41 / 237$ & 17.3 & 1.16 & $(0.58-2.33)$ & 1.03 & $(0.50-2.13)$ \\
\hline RIF mono-resistant TB & $15 / 109$ & 13.8 & 0.87 & $(0.39-1.97)$ & 0.65 & $(0.33-1.81)$ \\
\hline MDR-TB & $11 / 191$ & 5.8 & Ref & Ref & Ref & Ref \\
\hline \multicolumn{7}{|l|}{ HIV status at initiation } \\
\hline HIV negative & $1 / 61$ & 1.6 & 0.14 & $(0.02-1.01)$ & 0.13 & $(0.02-0.10)$ \\
\hline HIV positive on ART & $53 / 351$ & 33.6 & Ref & Ref & Ref & Ref \\
\hline HIV positive not on ART & $10 / 77$ & 12.1 & 1.49 & $(0.76-2.94)$ & 1.49 & $(0.75-2.96)$ \\
\hline HIV status unknown & $3 / 48$ & 6.3 & 0.38 & $(0.09-1.54)$ & 0.36 & $(0.09-1.50)$ \\
\hline \multicolumn{7}{|l|}{ Registration year } \\
\hline 2011 & $6 / 55$ & 10.9 & Ref & Ref & Ref & Ref \\
\hline 2012 & $11 / 108$ & 10.2 & 0.89 & $(0.33-2.40)$ & 0.88 & $(0.34-2.59)$ \\
\hline 2013 & $21 / 166$ & 21.7 & 1.3 & $(0.55-3.36)$ & 1.32 & $(0.52-3.33)$ \\
\hline 2014 & $29 / 211$ & 13.7 & 1.45 & $(0.59-3.51)$ & 1.37 & $(0.55-3.41)$ \\
\hline \multicolumn{7}{|l|}{ Time to treatment initiation } \\
\hline Without delay & $31 / 186$ & 16.7 & Ref & Ref & Ref & Ref \\
\hline Delayed (deferred) & $35 / 332$ & 10.5 & 0.89 & $(0.54-1.45)$ & 0.91 & $(0.53-1.56)$ \\
\hline
\end{tabular}

$H R$ Hazard Ratio, CI Confidence interval, RR-TB Rifampicin resistant TB, MTB Mycobacterium tuberculosis, MDR-TB Multi-drug resistant tuberculosis, XDR-TB Extensively drug-resistant tuberculosis; $A R T$ antiretroviral therapy, RIF rifampicin, DR-TB drug resistant TB $p<0.05$ bold

1448 new RR-TB patients who received treatment were recorded in the national register [2], but it may also reflect differences in the model of care. The majority (78\%; $540 / 686)$ of patients in our cohorts initiated treatment at a decentralized, outpatient DR-TB center, whereas the national cohort study included patients from both decentralized, outpatient and centralized, inpatient treatment facilities. The model of care has been shown to contribute to a decrease in time to treatment initiation, with outpatient sites demonstrating significantly lower time to treatment initiation compared to centralized, inpatient sites [3].

Pre-treatment mortality declined significantly from 17.5 to $5.8 \%$ between the early and late cohorts. As only $60.9 \%$ of patients in the late cohort were diagnosed using Xpert MTB/RIF, further expansion of Xpert MTB/RIF may make further reductions in pre-treatment mortality possible. Results suggest that the quality of tracing and success of linkage to care improved between the early and late periods of our study. The proportion of patients who were successfully traced and referred for treatment increased by $9 \%$, while the proportion of patients contacted and referred for treatment who failed to link to care in the expanded Xpert MTB/RIF cohort was roughly half of that observed during the early Xpert MTB/RIF cohort (17.8\% vs. 9.7\%). These tracing efforts may have translated into improved pre-treatment outcomes (i.e. more patients initiating appropriate DR-TB treatment and reducing mortality in the pre-treatment period). Not only was pre-treatment mortality significantly reduced between the early and late cohorts (17.5 to $5.8 \%$ ), but the time from sputum collection to death significantly increased from 19 to 29 days. The reduction in mortality may be associated with a reduction in time to treatment initiation observed during the late period. Time from specimen collection to the date a patient was considered lost to follow-up, untraceable, or transferred out of the city also increased from a median of 14 to 24 days. We speculate that tracing efforts improved during the late Xpert MTB/RIF period, causing the sickest 
patients to be diagnosed and started on treatment earlier while those who were less symptomatic failed to link to care and went on to die later.

Despite improved linkage to care and a reduction in pre-treatment mortality, pre-treatment loss to follow up remained high (17.5 and 22.4\%). Interventions such as the implementation of decentralized TB treatment and Xpert MTB/RIF thus do not appear sufficient to address alarmingly high rates of pre-treatment loss [3]. TB services could benefit from the implementation of a unique patient identifier so that health care workers can focus tracing efforts on patients who are "truly lost" and not waste efforts or resources on patients who have moved or transferred out of the city and initiated care at another facility, in the same or a different province.

We found no difference in on-treatment mortality ( $11.8 \%$ vs. $14.2 \%)$ and loss to follow-up ( $55.3 \%$ vs. $55.9 \%)$ when comparing the early and the late cohorts. Consistent with what others have reported, a reduction in time to treatment initiation did not result in improved treatment outcomes [18-24]. Prior studies have found no significant difference in on-treatment mortality between diagnosis by Xpert MTB/RIF and other diagnostic methods [8, 25-27]. As others have reported, despite reductions in time to treatment initiation, early treatment outcomes remain poor with high rates of death and loss from care [28].

HIV status was the only factor associated with mortality by 24 months. HIV negative patients were less likely to die compared to HIV-positive patients on ART (aHR 0.13 95\% CI 0.02-0.10). HIV prevalence in the late cohort was lower than has been described nationally among patients with rifampicin resistant TB $(47.1 \%$ vs. $66 \%)$ [2], but higher in the sub-analysis (79.7\%) as the data was more complete.

Results should be considered in light of the study limitations. First, Xpert MTB/RIF and decentralization of treatment in the COJ were rolled out in the same period. We cannot attribute our findings to either specific change, only to the combination of both. We did not have any data to explain differences in program performance during the early and late cohort periods. To address this, we allowed a 12-month period (July 2012 June 2013) between the two comparison cohorts to offset differences in program activity and resources as Xpert MTB/RIF and decentralized care were rolled-out across the different facilities. Second, the sub-analysis was limited to patients who initiated DR-TB treatment at one of the three decentralized outpatient DR-TB centers, thus potentially excluding those more likely to have worse outcomes (e.g. XDR, pre-XDR or those with poor clinical status). Third, treatment outcomes were missing for $37.2 \%$ of sub-study patients, and those without reported outcomes may have been more likely to have been lost to follow-up or died than those whose outcomes we observed. Data on HIV and ART status and time on ART were missing for patients who did not initiate DR-TB treatment. We also believe that among those with a diagnosis of RR-TB reported to the COJ, the proportion co-infected with HIV may be underestimated due to missing data. Nationally, for the period 2013, 13\% of patients with RR-TB had a missing HIV status, and this varied (9-22\%) across the nine provinces [2]. This is substantially lower than what we report (42\%) for the same period, and highlights the limitations of routine data. Some patients may have initiated treatment outside of the city, but existing tracing systems did not allow this to be determined. Finally, we speculate that mortality was underreported, as some patients who did not complete treatment and were reported as lost to follow-up or without an outcome likely died.

\section{Conclusion}

With South Africa's expansion of Xpert MTB/RIF coverage and treatment decentralization, both pre-treatment mortality and time to treatment initiation for DR-TB fell markedly. Xpert MTB/RIF resulted in an increase in the proportion of patients diagnosed with RR-TB who initiated treatment. These improvements did not, however, correspond to reductions in pre-treatment loss to follow-up or better DR-TB treatment outcomes and on-treatment mortality and loss to follow-up remained high.

Since rapid initiation of DR-TB treatment is key to controlling $\mathrm{TB}$, patients who are diagnosed with $\mathrm{TB}$ who do not initiate treatment (i.e. pre-treatment loss to follow-up) represent an important failing in the provision of care [6]. This group, who should be targeted because of their increased risk of mortality and TB transmission to others, are not included in routine reporting by national treatment programs, thus overestimating program effectiveness $[6,29]$. Programs should invest in novel ways to reduce pre-treatment loss to follow-up. Innovative approaches such as a mhealth linkage program or facility-based linkage officers should be explored to document pre-treatment loss to follow-up and improve linkage to care for DR-TB patients.

\section{Additional file}

Additional file 1: Figure S1. Total number of Xpert MTB/RIF tests performed between 2011 and 2014 (source: NHLS). (JPG 43 kb)

\section{Abbreviations}

aHR: Adjusted hazard ratio; aRR: Adjusted relative risk; ART: Antiretroviral therapy; COJ: City of Johannesburg; DR: Drug resistant; HIV: Human immunodeficiency virus; LPA: Line probe assay; MDR: Multi-drug resistant; MTB: Mycobacterium tuberculosis; NDOH: National Department of Health NHLS: National Health Laboratory Service; RIF: Rifampicin; RMR: Rifampicin mono-resistant tuberculosis; RR-TB: Rifampicin-resistant tuberculosis; 
TB: Tuberculosis; TTI: Time to treatment initiation; USA: United States of America; WHO: World Health Organization; XDR: Extensively drug resistant

\section{Acknowledgements}

The authors wish to thank the staff at all facilities, in particular for the support from the City of Johannesburg. A special thank you to Alice Kono, Busi Sithole, Portia Baloyi, Xolani Maseko, Antonia Barnard and Edwin Madibogo for all their help.

\section{Funding}

DE, TS, CG, RB and LL was supported through USAID agreement number \#674-A-12-00029 to the Health Economics and Epidemiology Research Office. KS and RB were supported by a cooperative agreement from the US Agency for International Development (USAID) to Right to Care \#674-A-1200020. AB was supported by USAID agreement number \#674-12-00002 to Wits Reproductive Health and HIV Research Institute. The contents of the article are the responsibility of the authors and do not necessarily reflect the views of USAID or the US government. The funders had no role in the study design, collection, analysis and interpretation of the data, in manuscript preparation or the decision to publish.

\section{Availability of data and materials}

The datasets generated and/or analysed during the current study are not publicly available as the data are owned by the study site (i.e. City of Johannesburg Metropolitan Municipality) and the National Department of Health (South Africa) and governed by the Human Research Ethics Committee (University of the Witwatersrand, Johannesburg, South Africa). All relevant data are included in the paper. The full data are available from the Health Economics and Epidemiology Research Office for researchers who meet the criteria for access to confidential data and with permission from the owners of the data. Contact the organization at information@heroza.org for additional information regarding data access.

\section{Authors' contributions}

Author Contributions: conceptualization: RB, KS, SR, AB. Author Contributions: methodology: KS, AB, RB. Author Contributions: software: $A B, R B, T S, C G, D E$. Author Contributions: validation: RB, KS, AB, SR, LL. Author Contributions: formal analysis: TS, CG, DE, KS. Author Contributions: investigation: CG, TS, KS, RB, DE, AB. Author Contributions: resources: SR, LL. Author Contributions: data curation: CG, TS, RB, DE, KS. Author Contributions: writing (original draft preparation): DE. Author Contributions: writing (review and editing): TS, RB, $\mathrm{KS}, \mathrm{AB}$, LL, SR. Author Contributions: visualization: SR, KS, RB, AB, LL. Author Contributions: supervision: RB, DE, LL, SR, KS. Author Contributions: project administration: DE, RB, SR, LL. Author Contributions: funding acquisition: LL, SR. All authors read and approved the final manuscript.

\section{Ethics approval and consent to participate}

The study was a retrospective register review of routinely captured records and a waiver of informed consent was granted to retrospectively review these records. Participants did not provide written or verbal consent to participate in the study as all data analysed were collected as part of routine diagnosis and treatment. This study and the analysis of anonymized data was approved by the Human Research Ethics Committee (Medical) of the University of the Witwatersrand (Wits HREC M130601).

\section{Consent for publication}

Not applicable

\section{Competing interests}

The authors declare that they have no competing interests.

\section{Publisher's Note}

Springer Nature remains neutral with regard to jurisdictional claims in published maps and institutional affiliations.

\section{Author details}

${ }^{1}$ Health Economics and Epidemiology Research Office, Department of Internal Medicine, School of Clinical Medicine, Faculty of Health Sciences, University of the Witwatersrand, Johannesburg, South Africa. ${ }^{2}$ Health Economics Unit, School of Public Health and Family Medicine, Faculty of Health Sciences, University of Cape Town, Cape Town, South Africa. ${ }^{3}$ Division of Infectious Diseases, University of North Carolina, Chapel Hill, NC, USA. ${ }^{4}$ Department of Internal Medicine, School of Clinical Medicine, Faculty of Health Sciences, University of the Witwatersrand, Johannesburg, South Africa. ${ }^{5}$ Department of Global Health, Boston University School of Public Health, Boston, MA, USA.

Received: 15 June 2018 Accepted: 23 November 2018

Published online: 17 December 2018

\section{References}

1. WHO. Global Tuberculosis Report. Geneva: World Health Organization; 2016. Available online http://www.who.int/tb/publications/global_report/gtbr15_ main_text.pdf. Accessed 01 April 2018. Accessed 11 May 2018

2. Cox H, Dickson-Hall L, Ndjeka N, Van't Hoog A. Delays and loss to follow-up before treatment of drug-resistant tuberculosis following implementation of Xpert MTB/RIF in South Africa: a retrospective cohort study. PLoS Med. 2017; 14(2):e1002238

3. Evans D, Schnippel K, Govathson C, Sineke T, Black A, Long L, Berhanu R, Rosen S. Treatment initiation among persons diagnosed with drug resistant tuberculosis in Johannesburg, South Africa. PLoS One. 2017;12(7):e0181238. https://doi.org/10.1371/journal.pone.0181238.

4. National Department of Health. 2011. Multi-drug resistant Tuberculosis: Policy framework on decntralized and deinstitutionalized management for South Africa. Available: http://www.tbfacts.org/wp-content/uploads/2015/ 08/SA-MDR-TB-Policy.pdf. Accessed 11 May 2018.

5. Cox HS, Daniels JF, Muller O, Nicol MP, Cox V, Van Cutsem G, Moyo S, De Azevedo V, Hughes J. Impact of decentralized care and the Xpert MTB/RIF test on rifampicin-resistant tuberculosis treatment initiation in Khayelitsha, South Africa. Open Forum Infect Dis. 2015;2(1):ofv014.

6. Macpherson P, Houben RM, Glynn JR, Corbett EL, Kranzer K. Pre-treatment loss to follow-up in tuberculosis patients in low- and lower-middle-income countries and high-burden countries: a systematic review and meta-analysis. Bull World Health Organ. 2014;92:26-38.

7. Meressa D, Hurtado RM, Andrews JR, Diro E, Abato K, Daniel T, Prasad P, Prasad R, Fekade B, Tedla Y, Yusuf H, Tadesse M, Tefera D, Ashenafi A, Desta G, Aderaye G, Olson K, Thim S, Goldfeld AE. Achieving high treatment success for multidrug-resistant TB in Africa: initiation and scale-up of MDR TB care in Ethiopia-an observational cohort study. Thorax. 2015;70(12):1181-8.

8. Mupfumi L, Makamure B, Chirehwa M, Sagonda T, Zinyowera S, Mason P, Metcalfe JZ, Mutetwa R. Impact of Xpert MTB/RIF on antiretroviral therapyassociated tuberculosis and mortality: a pragmatic randomized controlled trial. Open forum infectious diseases. 2014;1(1):ofu038.

9. Singla N, Satyanarayana S, Sachdeva KS, Van Den Bergh R, Reid T, TaylerSmith K, Myneedu VP, Ali E, Enarson DA, Behera D, Sarin R. Impact of introducing the line probe assay on time to treatment initiation of MDR-TB in Delhi, India. PLoS One. 2014;9:e102989.

10. Cox H, Dickson-Hall L, Jassat W, Moshabela M, Kielmann K, Grant A, Nicol M, Black J, Mlisana K, Vanleeuw L, Loveday M. Drug-resistant tuberculosis in South Africa: history, progress and opportunities for achieving universal access to diagnosis and effective treatment. SAHR - 20 Year Anniversary Edition; 2017. p. 157-68. Available online http://www.hst.org.za/ publications/South\%20African\%20Health\%20Reviews/15_Drug_ resistant\%20tuberculosis\%20in\%20South\%20Africa_history_ progress\%20and\%20opportunities.pdf. Accessed 01 Apr 2018

11. National Department of Health 2014. National Tuberculosis Guidelines 2014. National Department of Health Available online https://www.health-e.org. za/wp-content/uploads/2014/06/NTCP_Adult_TB-Guidelines-27.5.2014.pdf Accessed 01 Apr 2018

12. WHO 2010 TB treatment of TB guidelines 2010. 4th Ed. Geneva: World Health Organization. Available online http://apps.who.int/iris/bitstream/handle/10665/ 44165/9789241547833_eng.pdf;jsessionid= 5E5570095F7C77CB2371D60784ADD2B8? sequence=1. Accessed 27 Mar 2018.

13. World Health Organization. Definitions and reporting framework for tuberculosis. Geneva: World Health Organization; 2013. Available online http://apps.who.int/iris/bitstream/handle/10665/79199/9789241505345_eng. pdf?sequence=1. Accessed 11 May 2018

14. Schnippel K, Shearer K, Evans D, Berhanu R, Dlamini S, Ndjeka N. Predictors of mortality and treatment success during treatment for rifampicin-resistant tuberculosis within the south African national TB Programme, 2009 to 2011: a cohort analysis of the national case register. Int J Infect Dis. 2015;39:89-94. 
15. South African National AIDS Council. National strategic plan (NSP) for HIV and AIDS, TB and STIs 2012-2016. Pretoria: South African National AIDS Council; 2013. Available online https://heaids.org.za/site/assets/files/ 1267/national-strategic-plan-for-hiv-stis-and-tb-2012-2016.pdf. Accessed 01 Apr 2018

16. Cox H, Nicol MP. Tuberculosis eradication: renewed commitment and global investment required. Lancet Infect Dis. 2017;18(3):228-9.

17. GBD TB Collaborators. The global burden of tuberculosis: results from the global burden of disease study 2015. Lancet Infect Dis 2017. Available online http://dx.doi.org/10.1016/S1473-3099(17)30703-X. Accessed 14 May 2018.

18. Dheda K, Shean K, Zumla A, Badri M, Streicher EM, Page-Shipp L, Willcox P, John MA, Reubenson G, Govindasamy D, Wong M, Padanilam X, Dziwiecki A, Van Helden PD, Siwendu S, Jarand J, Menezes CN, Burns A, Victor T, Warren R, Grobusch MP, Van Der Walt M, Kvasnovsky C. Early treatment outcomes and HIV status of patients with extensively drug-resistant tuberculosis in South Africa: a retrospective cohort study. Lancet. 2010;375: 1798-807.

19. Harris RC, Grandjean L, Martin $\sqcup$, Miller AJ, Nkang JE, Allen V, Khan MS, Fielding $\mathrm{K}$, Moore DA. The effect of early versus late treatment initiation after diagnosis on the outcomes of patients treated for multidrug-resistant tuberculosis: a systematic review. BMC Infect Dis. 2016;16:193.

20. Jacobson KR, Barnard M, Kleinman MB, Streicher EM, Ragan EJ, White LF, Shapira O, Dolby T, Simpson J, Scott L, Stevens W, van Helden PD, Van Rie A, Warren RM. Implications of failure to routinely diagnose resistance to second-line drugs in patients with rifampicin-resistant tuberculosis on Xpert MTB/RIF: a multisite observational study. Clin Infect Dis. 2017:64(11):1502-8.

21. Kim DH, Kim HJ, Park SK, Kong SJ, Kim YS, Kim TH, Kim EK, Lee KM, Lee SS, Park JS, Koh WJ, Lee CH, Kim JY, Shim TS. Treatment outcomes and longterm survival in patients with extensively drug-resistant tuberculosis. Am J Respir Crit Care Med. 2008;178:1075-82.

22. Loveday M, Wallengren K, Voce A, Margot B, Reddy T, Master I, Brust J, Chaiyachati K, Padayatchi N. Comparing early treatment outcomes of MDRTB in decentralised and centralised settings in KwaZulu-Natal, South Africa. Int J Tuberc Lung Dis. 2012;16:209-15.

23. Mohr E, Cox V, Wilkinson L, Moyo S, Hughes J, Daniels J, Muller O, Cox H. Programmatic treatment outcomes in HIV-infected and uninfected drugresistant TB patients in Khayelitsha, South Africa. Trans R Soc Trop Med Hyg. 2015;109:425-32

24. Schnippel K, Firnhaber C, Ndjeka N, Conradie F, Page-Shipp L, Berhanu R, Sinanovic E. Persistently high early mortaity despite rapid diagnostics for drug-resitant tuberculosis cases in South Africa. Int J Tuber Lung Dis. 2017; 21(10):1106-11.

25. Churchyard GJ, Stevens WS, Mametja LD, McCarthy KM, Chihota V, Nicol MP, Erasmus LK, Ndjeka NO, Mvusi L, Vassall A, Sinanovic E, Cox HS, Dye C, Grant $A D$, Fielding KL. Xpert MTB/RIF versus sputum microscopy as the initial diagnostic test for tuberculosis: a cluster-randomised trial embedded in south African roll-out of Xpert MTB/RIF. Lancet Glob Health. 2015;3(8):e450-7. https:// doi.org/10.1016/S2214-109X(15)00100-X.

26. Auld AF, Fielding KL, Gupta-Wright A, Lawn SD. Xpert MTB/RIF - why the lack of morbidity and mortality impact in intervention trials? Trans R Soc Trop Med Hyg. 2016;1 10:432-44.

27. Cox HS, Mbhele S, Mohess N, Whitelaw A, Muller O, Zemanay W, Little F, Azevedo V, Simpson J, Boehme CC, Nicol MP. Impact of Xpert MTB/RIF for TB diagnosis in a primary care clinic with high TB and HIV prevalence in South Africa: a pragmatic randomised trial. PLoS Med. 2014;11(11):e1001760

28. Berhanu R, Schnippel K, Mohr E, Hirasen K, Evans D, Rosen S, Sanne I. Early outcomes of decentralized Care for Rifampicin-Resistant Tuberculosis in Johannesburg, South Africa: an observational cohort study. PLoS One. 2016; 11(11):e0164974. https://doi.org/10.1371/journal.pone.0164974.

29. Harries AD, Rusen ID, Chiang CY, Hinderaker SG, Enarson DA. Registering initial defaulters and reporting on their treatment outcomes. Int J Tuberc Lung Dis. 2009;13:801-3.

\section{Ready to submit your research? Choose BMC and benefit from}

- fast, convenient online submission

- thorough peer review by experienced researchers in your field

- rapid publication on acceptance

- support for research data, including large and complex data types

- gold Open Access which fosters wider collaboration and increased citations

- maximum visibility for your research: over $100 \mathrm{M}$ website views per year

At BMC, research is always in progress.

Learn more biomedcentral.com/submissions 